\title{
MicroRNA expression profiling of the fifth-instar posterior silk gland of Bombyx mori
}

\author{
Jisheng $\mathrm{Li}^{1,2+}$, Yimei Cai ${ }^{3+}$, Lupeng Ye ${ }^{1}$, Shaohua Wang ${ }^{1}$, Jiaqian Che ${ }^{1}$, Zhengying You ${ }^{1}$, Jun $\mathrm{Yu}^{3 *}$ and Boxiong Zhong ${ }^{1 *}$
}

\begin{abstract}
Background: The growth and development of the posterior silk gland and the biosynthesis of the silk core protein at the fifth larval instar stage of Bombyx mori are of paramount importance for silk production.

Results: Here, aided by next-generation sequencing and microarry assay, we profile 1,229 microRNAs (miRNAs), including 728 novel miRNAs and 110 miRNA/miRNA* duplexes, of the posterior silk gland the fifth larval instar. Target gene prediction yields 14,222 unique target genes from 1,195 miRNAs. Functional categorization classifies the targets into complex pathways that include both cellular and metabolic processes, especially protein synthesis and processing

Conclusion: The enrichment of target genes in the ribosome-related pathway indicates that miRNAs may directly regulate translation. Our findings pave a way for further functional elucidation of these miRNAs and their targets in silk production.
\end{abstract}

Keywords: MicroRNA, Silkworm, Posterior silk gland, Target gene

\section{Background}

The silkworm Bombyx mori is the most economically important holometabolous lepidopteran and has recently became an experimental model for molecular entomology $[1,2]$. Its silk gland is the most efficient protein synthesis machine among all organisms, which makes silkworm a desirable model for studying its mechanism. As the largest and most important part of the silk gland, the posterior compartment is most attractive since it synthesizes the silk core protein that determines the quality of silk cocoons. A recent proteomic study, using two-dimensional gel electrophoresis (2-DE) coupled with matrix-assisted laser desorption/ionization-time-of-flight mass spectrometry (MALDI-TOF MS), has identified 93 major proteins in the silk gland, of which there are several phosphorylated fibroin L-chain and P25 isoforms [3]. The posterior silk gland of the fifth instar has been further surveyed systematically for the understanding of molecular basis and regulatory mechanism of the posterior silk gland

\footnotetext{
* Correspondence: junyu@big.ac.cn; bxzhong@zju.edu.cn

${ }^{\dagger}$ Equal contributors

${ }^{3}$ Key Laboratory of Genome Sciences and Information, Beijing Institute of

Genomics, Chinese Academy of Sciences, Beijing 100029, China

${ }^{1}$ College of Animal Sciences, Zhejiang University, Hangzhou, Hangzhou

310058, P.R. China

Full list of author information is available at the end of the article
}

development and fibroin synthesis [4]. A recent transcriptomic survey has revealed a total of 10,393 active genes differentially expressed in multiple silkworm tissues on the third day of the fifth-instar larva, of which 412 and 109 are up-regulated in the anterior-middle and the posterior silk glands, respectively [5]. These findings all provide basic data for studying the growth of the posterior silk gland and fibroin synthesis. However, microRNAs (miRNAs)-based study has not been done for the silk gland and its developing and functionally important compartments albeit justifiable for necessity [6-8].

As a large family of endogenous small non-coding RNAs, miRNAs are common regulatory RNAs of eukaryotic organisms and play important roles in a wide range of biological processes under physiological and pathological conditions [9-14]. Despite lack of empirical data, computational approaches have made initial contributions to miRNA study in B. mori [15-18], followed by nextgeneration sequencing efforts that profiled miRNAs for different developmental stages and tissues [19-22]. Nevertheless, specifically focused study is still necessary since the expression of miRNAs is largely temporal-spatial [22-25].

Here, we report our miRNA profiling of the fifth-instar posterior silk gland, using next-generation sequencing and 
microarray technologies. We show that 728 out of 1,229 miRNAs are novel and 430 of the total are identified in the third day of the posterior silk gland development. Our GO (Gene Ontology)-based pathway assignment provides the first comprehensive categorization of B. mori miRNAs in the posterior silk gland.

\section{Results and discussion}

\section{Next-generation sequencing and data processing}

Rapid growth of the silk gland occurs at the fifth instar stage, and the gland is comprised of three distinct compartments: the anterior, the middle, and the posterior glands (Figure 1). Compared with the other two parts, the anterior gland, albeit smaller, serves as a duct to transport (spinning) silk proteins that form the cocoon. The middle gland produces considerable quantities of sericins and the longest posterior gland grows rapidly, synthesizing a series of proteins including fibroin heavy and light chains plus fibroin P25 by exclusively $\sim 500$ posterior gland cells of the fifth instar larva. As far as the biosynthesis of fibroin is concerned, the fifth instar stage can also be partitioned into two periods: the rapid formation and the massive secretion [26]. The third day of the fifth instar (V3) completes a division during larval development and rapid cell growth occurs at this period of time. Based on data from genome-scale expression profiling of the posterior silk gland, it has been concluded that gene expression profile from the fourth instar molting to the fifth instar day 8 before spinning forms two clusters that is divided at V3 from the fourth molting to wandering periods [4]. A large amount of genes encoding the fibroin light chain, fibrohexamerin P25, transcription factors, structural proteins, glucose and other sugar transporters and proteins that aid in hormone signal transduction are up-regulated in the posterior silk gland from V1 to V5, and are slightly downregulated at the wandering stage $[4,5]$. Therefore, changes of gene expression at the fifth instar may be responsible for growth and development of the posterior silk gland, especially various miRNAs that play regulatory roles in post-transcriptional control [27].

The raw and processed data of all samples have been deposited in NCBI's Gene Expression Omnibus (GEO) [28] under accession number GSE 56380. From 93.2 million processed reads ranging from $18 \sim 30$ nt in length (Table 1, Additional file 1: Figure S1), we first examined the length distribution of small RNAs from ten libraries and found two extremely high peaks in most libraries; one was around $20 \mathrm{nt}$ and the other around $28 \mathrm{nt}$ (Additional file 2: Figure S2). This result is consistent with the previous reports, where the first peak was proposed to represent miRNAs and the other was interpreted as longer piRNAlike small RNAs [21,22,29,30]. We subsequently categorized non-coding small RNAs and defined them according to Rfam database 10.0. The individual expression level of small RNAs is very similar across the 10 libraries (Additional file 3: Table S1). We found that rRNAs and tRNAs were the majority of all non-coding RNA categories, as they are accounted for the most components of protein synthesis.

\section{Known and novel miRNAs based on sequence data}

After the removal of larger RNAs, we mapped the remaining reads (18-30 nt) to miRBase 16.0 [31] using the deep-sequencing small RNA analysis pipeline (DSAP). DSAP is a fast web server specially designed to analyze known miRNAs generated from the Illumina sequencing platform and yields satisfactory results [32,33]. Our effort yielded 304 known miRNAs (Additional file 4: Table S2), accounted for a large proportion of miRBase 16.0 (http:// www.mirbase.org/cgi-bin/browse.pl), which are 20-27 nt in size and have the highest abundance $(71.38 \%)$ in a range of 21-23 nt. We grouped them into 66 miRNA families except for some undefined miRNAs (Additional file 4: Table S2 and Additional file 5: Figure S3). Based on cross-species analysis, these known miRNAs are shared

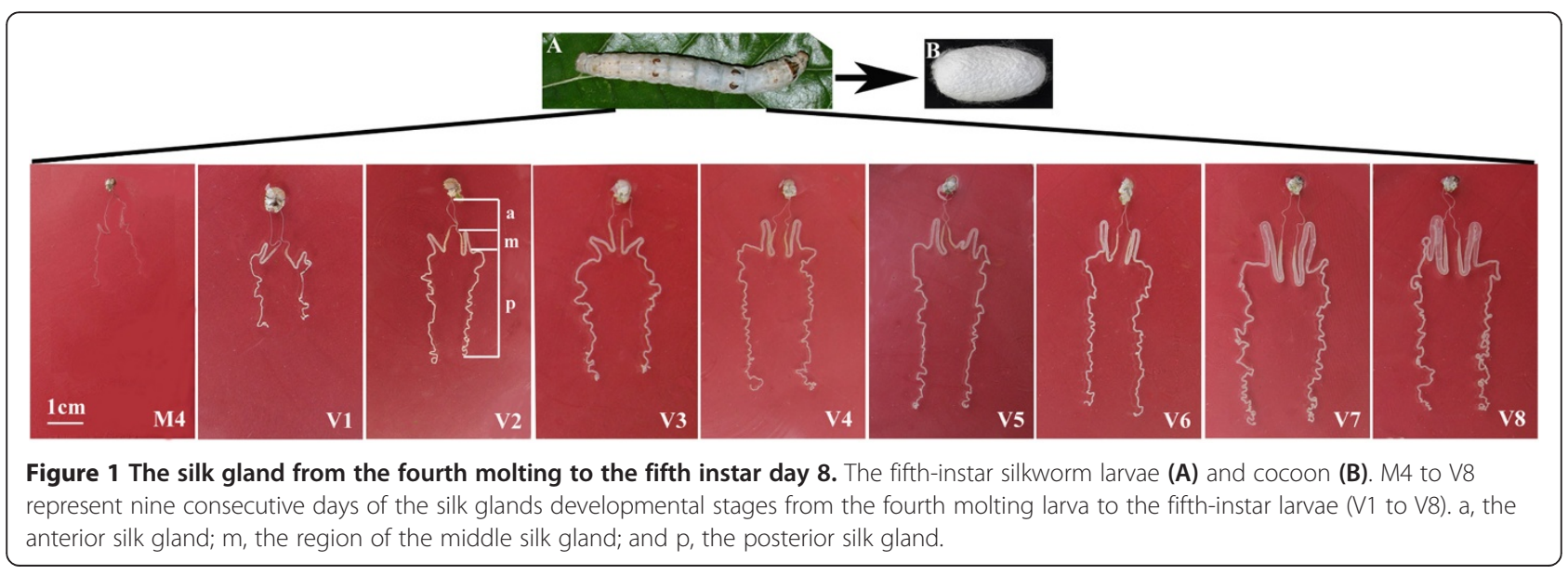


Table 1 Data summary of sequenced small RNAs based on DSAP

\begin{tabular}{lcccccccccc}
\hline & Q1 & Q2 & B1 & B2 & QB1 & QB2 & BQ1 & BQ2 & R1 & J1 \\
\hline Total reads & 18707473 & 18633295 & 18556476 & 18499427 & 17947940 & 16942708 & 17449851 & 17905942 & 10508573 & 12079631 \\
Cleaned sequence tags & 901684 & 674947 & 810182 & 622202 & 633421 & 617616 & 857114 & 617471 & 1162861 & 816340 \\
Reads in cleaned sequence tags & 10044506 & 10693830 & 10790311 & 9085018 & 7461204 & 8113977 & 9405884 & 9159736 & 8614699 & 9822808 \\
\% reliable reads \# & 53.69 & 57.39 & 58.15 & 49.11 & 41.57 & 47.89 & 53.90 & 51.15 & 81.98 & 81.32 \\
Unique Sequence Clusters (USC) & 901684 & 674947 & 810182 & 622202 & 633421 & 617616 & 857114 & 617471 & 1162861 & 816340 \\
Matched ncRNA in Rfam & 690 & 608 & 673 & 596 & 642 & 569 & 701 & 602 & 739 & 693 \\
USC matched to Rfam & 134580 & 97673 & 122849 & 105997 & 89704 & 92104 & 127959 & 101047 & 154898 & 145107 \\
Reads matched to Rfam & 2729256 & 2440852 & 2908825 & 2573860 & 1644409 & 2172466 & 2133486 & 2565135 & 1108916 & 1386447 \\
\% reads matched to Rfam & 27.17 & 22.82 & 26.96 & 28.33 & 22.04 & 26.77 & 22.68 & 28 & 12.87 & 14.11 \\
Matched miRNAs in miRBase & 181 & 170 & 182 & 157 & 181 & 150 & 202 & 168 & 239 & 220 \\
USC matched to miRBase & 1411 & 1167 & 1216 & 927 & 1174 & 817 & 1643 & 1016 & 2540 & 2089 \\
Reads matched to miRBase & 406154 & 744285 & 540458 & 277369 & 486835 & 339576 & 544337 & 310026 & 1486269 & 1576622 \\
\% reads matched to miRNAs & 4.04 & 6.96 & 5.01 & 3.05 & 6.52 & 4.19 & 5.79 & 3.38 & 17.25 & 16.05 \\
USC Unmatched reads & 765693 & 576107 & 686117 & 515278 & 542543 & 524695 & 727512 & 515408 & 1005423 & 669144 \\
\hline
\end{tabular}

Note: \#Percentage of reliable reads $=$ (Number of reads in cleaned sequence tags/Number of total reads)* 100.

by $\sim 68$ species. Among them, 40 families are widely conserved in insects and 26 families are unique to $B$. mori. Moreover, 25 families are distributed among 14 classes/phyla including both invertebrates and vertebrates (Figure 2 and Additional file 6: Figure S4).

The read count for different miRNA is rather variable (Additional file 4: Table S2). For instance, the number of reads for bmo-miR-263a is extremely high as compared to other miRNAs in all libraries; it may play a very important role in the posterior gland development and the result is in agreement with a previous report [20]. We also found 50 pairs of miRNA/miRNA* duplexes, in addition to 24 miRNA*s without the corresponding miRNAs. Although most miRNAs are more abundant than their corresponding miRNA*s, there are exceptional cases, where bmo-miR-10, bmo-miR-276, bmo-miR-305, bmo-miR-33, and bmo-miR-34 are less abundant than their miRNA* counterparts. Similar findings have also been reported in other deep sequencing experiments and are suspected to be a result of incorrect annotations in miRBase [34-36].

Having filtered the known non-coding RNAs, we predicted novel candidate miRNAs using the mireap package [37] and classified 1,427 candidate miRNAs (Additional file 7: Table S3). Given the fact that there are many random inverted repeats (termed pseudo-hairpins) in eukaryotic genomes and they can also fold into dysfunctional hairpins and undistinguished sequences, we took extra cautions to classify non-conserved miRNAs. We used mirident classifier to identify the miRNA candidates, which has been reported to achieves $99.2 \%$ specificity and $97.6 \%$ sensitivity on a human test data set [38]. We also evaluated two other SVM-based prediction programs, Triplet-SVM and PmirP, together with mirident and using miRbase datasets that include data from 24 insect species [38-40]. Mirident classifier gave rise to better results for insect premiRNA identification in our own hands [41]. Using mirident classifier, we obtained 613 novel miRNAs, corresponding to 590 unique sequences after filtering pseudopre-miRNAs (Additional file 7: Table S3).

\section{Microarray-based miRNA profiling}

Since the third day of fifth-instar larva (V3) is a key time point for silk synthesis and rapid cell growth, we evaluated its miRNA expression profile using 3,077 custom-designed probes (Additional file 1: Figure S1 and Additional file 8: Table S4) that are classified into four group: (1) 1,006 known miRNAs from miRBase, which consist of miRNAs from several species, including silkworm (559 probes) and 10 flies (447 probes); (2) 1,427 predicted novel miRNAs; (3) 425 probes based on data from four publications $[17,19,22,42]$; and (4) 219 control sequences. To ensure reproducibility, we double-gridded 841 sequences with read coverage of $>5$ from 1,427 custom-designed probes for each chip.

We used 16 chips (probe sets) for the study and normalized the data in $\log 2$ transformation. Both technical (all $\mathrm{R}^{2}>0.97$ ) and biological repeats (all $\mathrm{R}^{2}>0.8$ ) showed consistent results (Additional file 9: Figure S5 and Additional file 10: Table S5). As miRNA-based microarray experiments in general are reproducible [43-45], we readily identified 430 mature miRNAs. Among them, 239 are previously known and the remaining 191 include 19 conserved in Drosophila and 172 novel ones (Additional file 11: Table S6). Of the 239 known miRNAs, 187 are from a thorough collection from literature search and the 


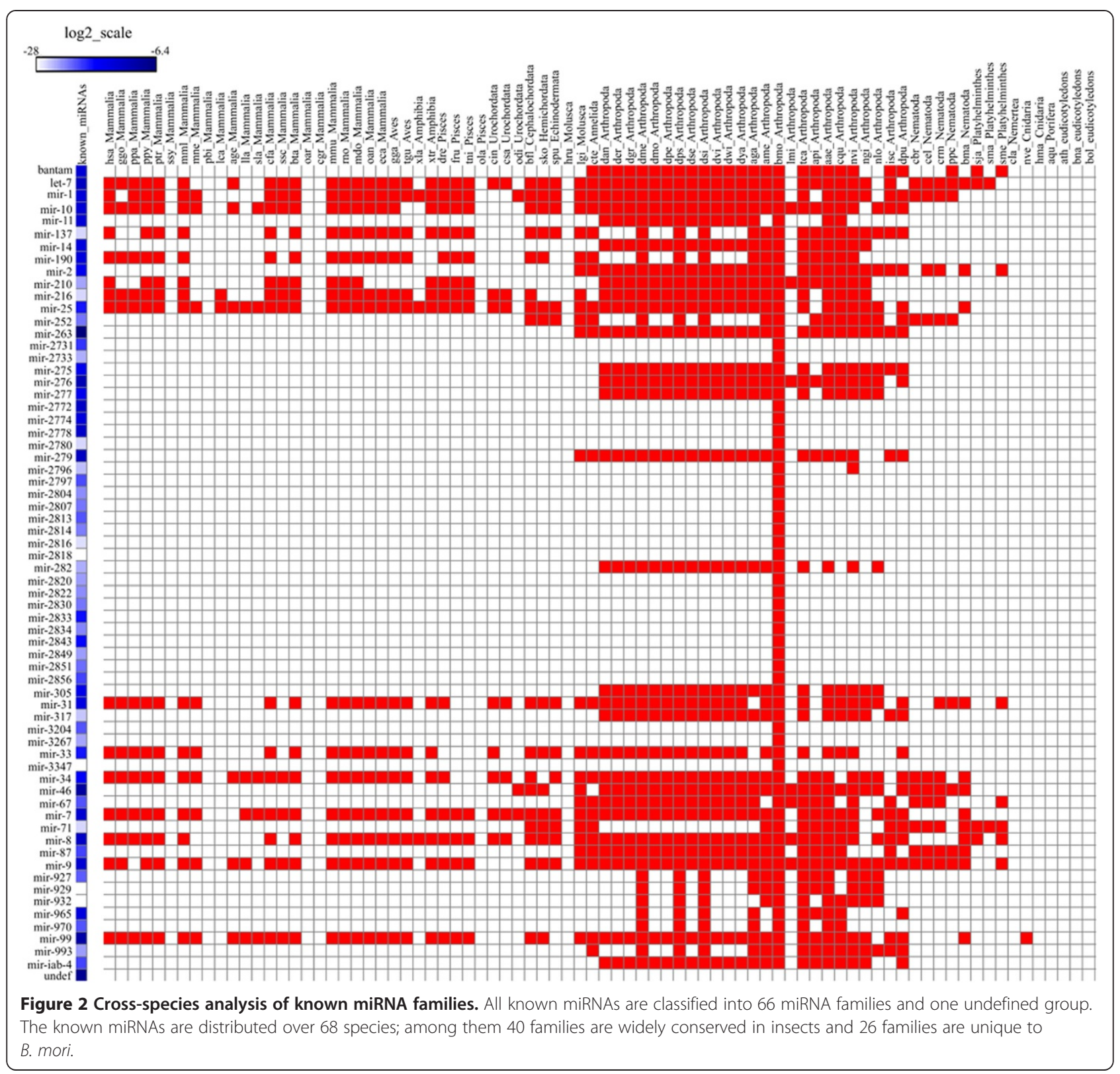

remaining 52 are directly from the miRBase. These miRNAs showed different expression patterns among the samples and did not exhibit any obvious correlation to information sources. For instance, bmo-bantam, bmomiR-12, bmo-miR-263a, bmo-miR-263b, bmo-miR-278, and bmo-miR- 8 are both literature-based and database collections but the miRBase-collected miRNAs showed stronger signals. The contrary results were found among bmo-let-7, bmo-miR-1, bmo-miR-100, bmo-miR-124, bmo-miR-137, bmo-miR-14, bmo-miR-252, bmo-miR275, bmo-miR-305, bmo-miR-307, bmo-miR-34, and bmomiR-279c, where the literature-based collections showed higher expression levels (Additional file 12: Table S7). We inferred that this non-uniformity might be a result of different technical platforms. One discussion point on this study is the validation rate: only 172 novel miRNAs ( 14\%) from the sequencing data are confirmed in the microarray experiment. The reasons for such low confirmation are multifold. First, 430 mature miRNAs are detected on the third day of fifth instar but not on the entire stage, where samples are collected and pooled from the fourth instar molting to the fifth instar day 8 before spinning. The false negative results for some of the miRNAs are largely due to the dilution of the timesensitive specific miRNAs over pooling $[46,47]$. Second, the marginal level of miRNA expression is pushing the detection limit so that some of the signals may not be consistently detected even when the same experiments 
are repeated. Third, sampling bias may be inherent from the sequencing approach, where sampling bias is obvious for low copy transcripts $[27,43]$.

There are 257 miRNA genes whose expression patterns have been reported to correspond to 324 loci in the $B$. mori genome [22]. After the removal of redundant sequences, we found that the two datasets shared 197 miRNAs (16 sequences showing discrepancy in their sequences were not accounted for). Among these miRNAs, 75 genes showed posterior gland expression in the current study but have not been detected in the previous study. Conversely, from 173 miRNAs identified previously in two public datasets for posterior-silk-gland expression, 37 were negative in our study. In addition, seven (bmo-miR-2846, bmo-miR-2850a, bmo-miR-2853, bmo-miR-2854, bmo-miR-2858*, bmo-miR-2858, and bmo-miR-2859) out of 21 posterior-gland specific miRNAs defined previously were not detected in our microarray experiment (Additional file 13: Table S8). These results suggest that our experiment covered most of the $B$ mori miRNAs but inconsistency does exist, attributable to the difference between technical platforms.

\section{Target gene prediction and pathway analysis}

Combining results from both deep sequencing and microarray, we identified 1,229 miRNAs expressed in the posterior silk gland in the period of the fourth-instar molting to the fifth-instar (day 8 before) spinning, and among which 728 are novel, named as bmo-miR-Pxxx-xp series (from No.1 to No.728 at Additional file 14: Table S9), and 110 are miRNA/miRNA* duplexes (Additional file 15: Table S10). We also profiled 430 miRNAs for the third day of the fifth-instar larva (Additional file 11: Table S6). We subsequently predicted potential targets using miRanda v3.3a [48] and the effort yielded 14,222 targets in the entire stage, corresponding to 1,195 miRNAs. The rest 34 miRNAs did not yield target genes due to low scores (Additional file 16: Table S11). We associated 12,675 and 12,948 target genes to 423 known and 696 novel miRNAs in the third day of the fifth instar, respectively (Additional file 17: Table S12 and Additional file 18: Table S13).

We annotated all miRNA target genes based on GO analysis and found that they are largely involved in cell, cell part, binding, catalytic, cellular process, metallochaperone, proteasome regulator, and metabolic process, as opposed to the underrepresented synapse, synapse part, and viral reproduction (Figure 3). This result suggests that miRNAs may regulate mostly the expression of structural protein genes in the posterior gland than those involved in the development of neural and immune systems. Furthermore, our GO analysis on the target genes of novel miRNAs and those detected at the third day of the fifth instar showed a similar result to that of the total miRNAs, except the absence of viral reproduction in biological process terms and metallochaperone in molecular function terms. Finally, as the major silk protein secretary organ, the posterior silk gland has an increased ribosome content $[4,49,50]$, and it has been reported that ribosomal proteins are abundantly expressed in the final instar and play key roles in modulating activity of ribosome $[49,51]$. Our result confirmed this observation.

Based on further comparison of biological pathways among three datasets (the entire stage, the novel miRNAs, and the V3 group), we showed that 5,871 out of 14,222 predicted targets in the entire stage were involved in 302 KEGG pathways (Figure 4, Additional file 19: Table S14). The other two sets of target genes shared a similar result, where 5,400 and 5,331 target genes from the novel miRNAs and V3 group were mapped onto these pathways, respectively (Additional file 19: Table S14; Figure 5). Furthermore, there were 107 target genes mapped to the ribosome pathway (Figure 6, Additional file 20: Figure S6 and Additional file 19: Table S14) and 92 target genes involved in protein processing of endoplasmic reticulum pathway in the entire stage (Additional file 21: Figure S7 and Additional file 19: Table S14). Since translation-level regulation of ribosomal proteins is critical for fibroin synthesis [4], most of the target genes (107, 96 and 93 in the entire stage, the novel miRNA, and the V3 group, respectively) were mapped to ribosome pathway for all three datasets as compared to other pathways, and these target genes almost covered all the genes in this pathway (Figure 5, Figure 6 and Additional file 20: Figure S6). These results indicate that miRNAs expressed in the fifth instar of posterior silk gland showed strong regulatory functions on the silk protein synthesis.

Other involved pathways are also informative. First, 99 target genes are related to RNA transport pathway, and 47 target genes are mapped to RNA degradation pathway (Additional file 19: Table S14 and Additional file 22: Figure S8). Nearly 90 and 50 target genes are involved in purine and pyrimidine metabolisms, respectively (Additional file 19: Table S14, Additional file 23: Figure S9 and Additional file 24: Figure S10 ). The results indicate active regulations of transcription and nucleotide metabolism. Second, 79 target genes are found to be involved in oxidative phosphorylation pathway (Additional file 19: Table S14 and Additional file 25: Figure S11). The ATP production pathway may coordinate with nucleotide metabolic pathways for energy generation. Third, 66 and 33 target genes are related to ubiquitin mediated proteolysis and proteasome pathways, respectively (Additional file 19: Table S14 and Additional file 26: Figure S12). Since ubiquitin proteolytic system plays an important role in a broad array of basic cellular processes including regulation of cell cycle, modulation of the immune and inflammatory responses, control of signal transduction 


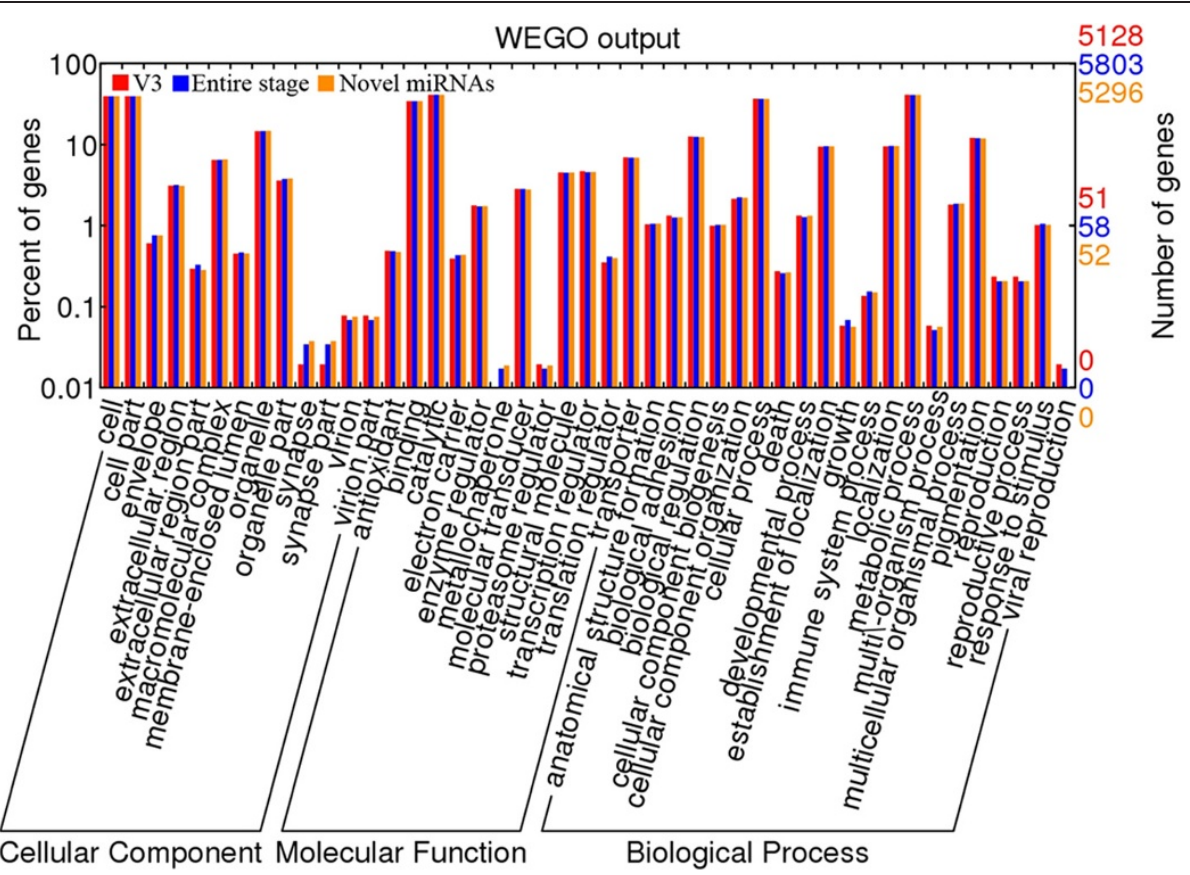

Figure $3 \mathrm{GO}$ analyses of the target genes predicted by miRanda in the posterior silk gland. We analyzed three groups of miRNAs: (1) V3, 430 miRNAs detected at the third day of fifth instar; (2) Entire Stage, 1,229 miRNAs discovered at the periods from the fourth instar molting to the fifth instar day 8 before spinning, and (3) Novel, 728 miRNAs first detected in this study.

pathways, development and differentiation, and these complex processes are controlled by specific degradation of a single or a subset of proteins [52], the discovery of such a significant involvement is of importance. Fourth, we observed 64 target genes mapped to cell cycle pathway (Additional file 19: Table S14 and Additional file 27: Figure S13) which suggests that these miRNAs may be regulators of cell cycle. It has been well established that cell division only occurs during the embryonic development, and the number of cells in the posterior silk gland no longer increases throughout the larval life [53]. Finally, pathway analysis results showed highly consistency between the three datasets: the entire stage, the novel miRNAs, and the V3 group.

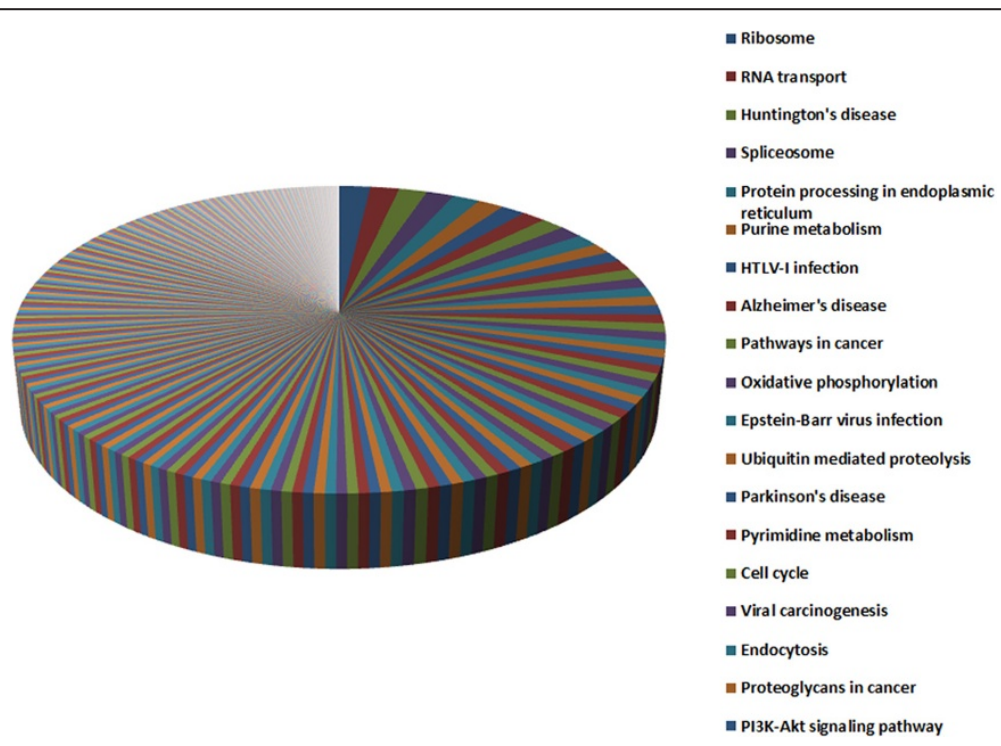

Figure 4 The number of miRNA target genes mapped on pathway. The miRNAs detected at Entire stage from the fourth instar molting to the fifth instar day 8 before spinning. 


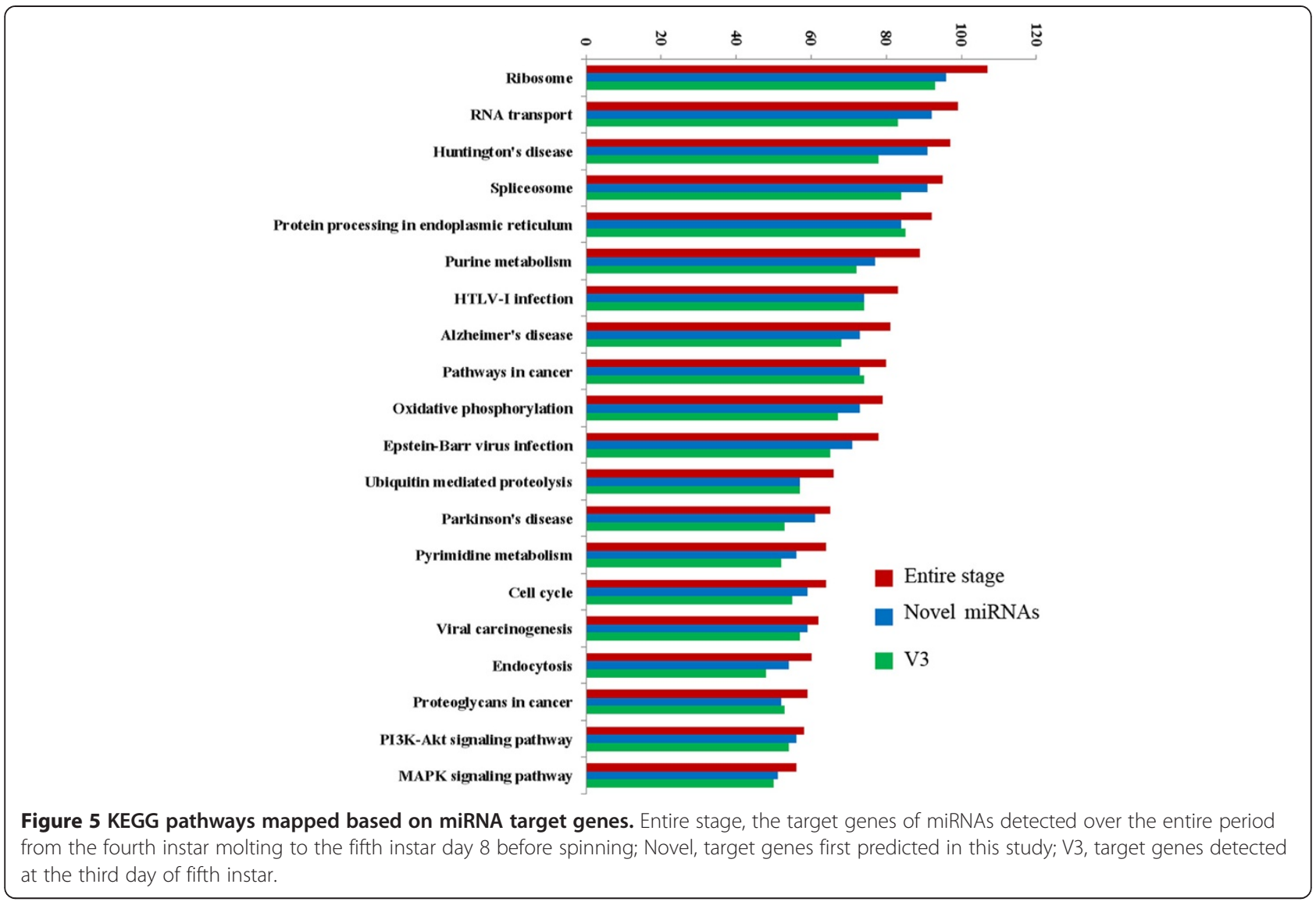

\section{Conclusion}

From 10 small RNA libraries, we acquired $\sim 93$ million processed reads ranging from $18-30 \mathrm{nt}$ in length, identified 1,229 miRNAs (110 miRNA/miRNA* duplexes), and profiled 430 miRNAs at the third day of the fifth instar larva. We also found 728 novel miRNAs (including 55 miRNA/miRNA* duplex and $709 \mathrm{Bm}$-specific miRNAs [54]. Our findings expanded the collection of B. mori miRNAs in miRBase and covered most miRNAs of the posterior silk gland. Moreover, on the discovery of target genes [51-53], we predicted 14,222 targets matching 1,195 miRNAs, which are classified into many important pathways including protein synthesis, energy supply, and cell cycle control. Our results underscore the key regulatory roles that miRNAs play in the fifth instar posterior silk gland for silk production.

\section{Methods}

\section{Silkworm rearing and sample preparation}

For better miRNA profiling and eliminating strain-specific effects, we selected six domesticated silkworm strains (Q, Qiufeng; B, Baiyu; QB, Qiufeng $\times$ Baiyu; and BQ, Baiyu $\times$ Qiufeng, R1, and J1) and reared them on fresh mulberry leaves under standard condition. We used three sets of samples according to genes expression cluster analysis [4]: (1) Stage 1: fourth instar molting to day 2 of fifth instar from Q, B, QB, and BQ); (2) Stage 2: fifth instar day 3 to day 8 before spinning; and (3) Entire stage: Stage $1+2$ from R1 and J1. The samples were collected daily and dissected and stored at low temperature in $0.7 \% \mathrm{NaCl}$. Samples were subsequently rinsed and stored in liquid nitrogen.

\section{Small RNA library construction and solexa sequencing}

Total RNA was extracted from the posterior silk gland with Trizol reagent (Invitrogen, Carlsbad, CA, USA) according to the manufacturer's instructions. For miRNAseq, total RNA of the desired size range (18-30 nt) was size-fractionated on a $15 \%$ PAGE gel and ligated with to sequence adapters (T4 RNA ligase). After amplified for $15 \mathrm{cy-}$ cles, the products were separated on agarose gels and the RT-PCR products were sequenced on the Illumina platform (Beijing Genomics Institute or BGI, Shenzhen) $[55,56]$.

\section{Sequence analysis and microRNA prediction}

Raw sequence reads of $35 \mathrm{nt}$ in size were generated and unique reads of full-length small RNA sequences $(\geq 18 \mathrm{nt})$ were analyzed with deep sequencing small RNA analysis pipeline (DSAP) (http://dsap.cgu.edu.tw/dsap.html). Unique reads matching silkworm non-coding RNA (rRNA. tRNA, 

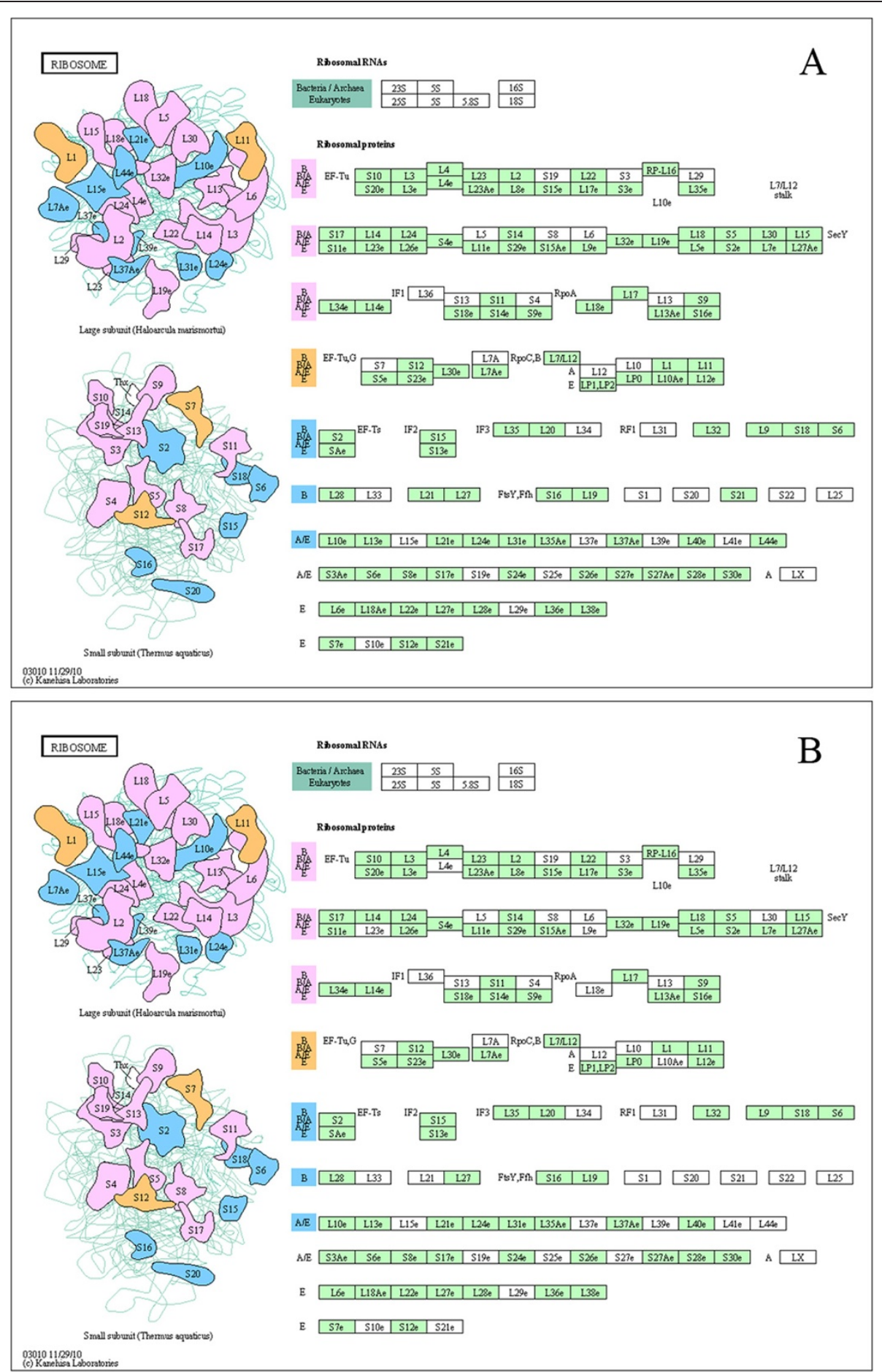

Figure 6 The ribosome pathway. miRNA target genes detected in the entire period from the fourth instar molting to the fifth instar day 8 before spinning (A) and target genes first detected in this study (B). Mapped pathways were highlighted in green.

sRNA, snoRNA and other non-coding RNA) deposited at the NCBI GenBank database and Rfam 10.0 were removed. The clean reads from raw dataset were matched to the known miRNA in miRBase 16.0 (http://microrna. sanger.ac.uk) to identify conserved miRNAs and annotated stem-loop sequences. After strict screening, the remaining sequences were regarded as candidate miRNAs for further analysis. 
To determine potential novel miRNAs, we identified candidate miRNAs using the mireap program (http://sourceforge.net/projects/mireap), which is an algorithm developed by BGI, which can be used to identify known miRNAs and novel candidates with canonical hairpin structure and sufficiently supported by sequencing data. In the present study, mireap parameters were set to match the condition of animal miRNAs identification as follows: (1) the length range of the miRNA sequence: 20-24 nt; (2) the maximal free energy allowed for an miRNA precursor: $-18 \mathrm{kcal} / \mathrm{mol}$; (3) the minimal common base pairs between miRNA and miRNA*: 14 with no more than four bulges; and (4) the maximal asymmetry of miRNA/miRNA* duplex: 5 nt. Following miRNA prediction, secondary structures were predicted by using the Zuker algorithm that evaluates hairpin forming potential (http://rna.urmc.rochester.edu/rnastructure.html).

\section{Microarray analysis}

To determine comprehensive miRNA expression profiles on the third day of fifth instar larvae, we collaborated with LC Bio Co. Ltd (LC sciences, USA) developed and designed miRNA probes. Considering that miRNA expression profiles may vary in different varieties and genders [46], we collected both male and female silkworms from four stains $(\mathrm{Q}, \mathrm{B}, \mathrm{QB}$, and $\mathrm{BQ})$ in duplicates. The small RNA fraction was extracted with Trizol reagent (Invitrogen, Carlsbad, CA, USA). To ensure the quality of the RNA, we used checked RNA quality and quantity with spectrophotometer and size-fractionated it using YM-100 Microcon centrifugal filter (Millipore). After adding poly-A tails, hybridization (10 $\mu \mathrm{g}$ probe) was used was carried out on a $\mu$ Paraflo $^{\text {тм }}$ microfluidic chip (Atactic Technologies) [57]. After imagine acquisition (GenePix 4000B, Molecular Device; Media Cybernetics) and background removal, we normalized the signals using a LOWESS (Locally-weighted Regression) method [58], classified the data using a hierarchical clustering method and average linkage and Euclidean distance metric, and visualized the results with TIGR MeV (Multiple Experimental Viewer; Institute for Genomic Research).

\section{Target gene prediction analysis}

Due to lack of available 3'-utr database, we first estimated the unigenes from NCBI (release date: Mar 30, 2006) and considered $1 \mathrm{~kb}$ as a suitable length for silkworm 3'-utr. Then, according to the annotation of silkdb2.0 (http:// www.silkdb.org/silkdb), $1 \mathrm{~kb}$ sequences after the last exon of annotated genes were selected as target gene region. Finally, we used miRanda v3.3a (http://cbio.mskcc. org/microrna_data/manual.html) to predict potential targets. The thresholds for candidate target sites were set at $\mathrm{S} \geq 140$ and $\Delta \mathrm{G}<-20 \mathrm{kcal} / \mathrm{mol}[48]$.

\section{Gene ontology and KEGG pathways analysis}

We analyzed the function of miRNA targets based on Gene Ontology through searching against InterPro and KEGG databases (http://www.genome.jp/kegg/), using InterProScan, WEGO (http://wego.genomics.org.cn/), and UniProtKB (http://pir.georgetown.edu/pirwww/search/ blast.shtml).

\section{Additional files}

\section{Additional file 1: Figure S1. Step-by-step workflow of the strategy for} posterior silk gland of silkworm miRNA expression profiling analysis.

Additional file 2: Figure S2. Length distribution of small RNAs from 10 libraries of posterior silk gland of silkworm from the Illumina data.

Additional file 3: Table S1. Rfam expression level of ten libraries of posterior silk gland of silkworm

Additional file 4: Table S2. Known miRNAs detected of posterior silk gland of silkworm.

Additional file 5: Figure S3. The length distribution of all known miRNAs summarized by DSAP.

Additional file 6: Figure S4. Phylogenic distribution analysis of known miRNA families. Similar to Figure 3, we find 25 families are distributed in over 14 classes or phylums from invertebrates to vertebrates according to phylogenic distribution.

Additional file 7: Table S3. Candidate novel miRNAs predicted by mireap program and identified by mirident classifier and miRAlign.

Additional file 8: Table S4. The composition of probes in microarray assay.

Additional file 9: Figure S5. Biological repeats correlation analysis. In order to verify the reliability of microarray assay, male $(M)$ and female (F) silkworms of four stains were separately treated and repeated twice. Q stands for Qiufeng and B represents Baiyu, while QB and BQ are their reciprocal cross breeds.

Additional file 10: Table S5. Significance testing for correlation of technical repeats by probes of repeated twice.

Additional file 11: Table S6. miRNAs of posterior silk glands confirmed by microarray assay.

Additional file 12: Table S7. Comparison miRNAs expression level with miRBase.

Additional file 13: Table S8. Comparison of miRNAs of the posterior silk glands with previous report.

Additional file 14: Table S9. Total and novel miRNAs detected in silkworm posterior silk gland.

Additional file 15: Table S10. All miRNA/miRNA* duplex detected in silkworm posterior silk gland.

Additional file 16: Table S11. Target genes of miRNAs detected at the entire stage prediction using miRanda.

Additional file 17: Table S12. Target genes of miRNAs detected at the third day of the fifth instar prediction using miRanda.

Additional file 18: Table S13. Target genes of novel miRNAs prediction using miRanda.

Additional file 19: Table S14. The number of posterior silk gland miRNA target genes mapped on pathway.

Additional file 20: Figure S6. The ribosome pathway. Target genes of the third day of the fifth instar detected miRNA. Mapped pathways were highlighted in green.

Additional file 21: Figure S7. The oxidative phosphorylation pathway. (A) miRNA target genes detected in the entire period from the fourth instar molting to the fifth instar day 8 before spinning, (B) target genes first detected in this study; and (C) target genes detected in the third day of the fifth instar. Mapped pathways were highlighted in green. 
Additional file 22: Figure S8. The purine metabolism pathway. (A) miRNA target genes detected in the entire period from the fourth instar molting to the fifth instar day 8 before spinning, (B) target genes first detected in this study; and (C) target genes detected in the third day of the fifth instar. Mapped pathways were highlighted in green.

Additional file 23: Figure S9. The pyrimidine metabolism pathway. (A) miRNA target genes detected in the entire period from the fourth instar molting to the fifth instar day 8 before spinning, (B) target genes first detected in this study; and (C) target genes detected in the third day of the fifth instar. Mapped pathways were highlighted in green.

Additional file 24: Figure S10. The RNA transport pathway. (A) miRNA target genes detected in the entire period from the fourth instar molting to the fifth instar day 8 before spinning, (B) target genes first detected in this study; and $(C)$ target genes detected in the third day of the fifth instar. Mapped pathways were highlighted in green.

Additional file 25: Figure S11. The cell cycle pathway. (A) miRNA target genes detected in the entire period from the fourth instar molting to the fifth instar day 8 before spinning, (B) target genes first detected in this study; and (C) target genes detected in the third day of the fifth instar. Mapped pathways were highlighted in green.

Additional file 26: Figure S12. The ubiquitin mediated proteolysis pathway. (A) miRNA target genes detected in the entire period from the fourth instar molting to the fifth instar day 8 before spinning, (B) target genes first detected in this study; and (C) target genes detected in the third day of the fifth instar. Mapped pathways were highlighted in green.

Additional file 27: Figure S13. The protein processing in endoplasmic reticulum pathway. (A) miRNA target genes detected in the entire period from the fourth instar molting to the fifth instar day-8 before spinning, (B) target genes first detected in this study; and (C) target genes detected in the third day of the fifth instar. Mapped pathways were highlighted in green.

\section{Abbreviations}

miRNA: microRNA; 2-DE: two-dimensional gel electrophoresis; MALDI-TOF MS: Matrix-assisted laser desorption/ionization-time-of-flight mass spectrometry; GO: Gene ontology; KEGG: Kyoto encyclopedia of genes and genomes; DSAP: Deep-sequencing small RNA analysis pipeline; Q: Qiufeng; B: Baiyu; QB: Qiufeng $\times$ Baiyu; BQ: Baiyu × Qiufeng.

\section{Competing interests}

The authors' declare that they have no competing interests.

\section{Authors' contributions}

$J L$ performed the statistical analysis, drafted and revised the manuscript. YC carried out the bioinformatics analysis and revised the manuscript, LY, SW, JC and $Z Y$ reared and harvested all the samples, isolated small RNAs, edited figures and additional files, and revised the manuscript. JY participated in the design of the study and helped to draft and revised the manuscript. BZ conceived and coordinated the study, and revised the manuscript. All authors read and approved the final manuscript.

\section{Authors' information}

JL: 'College of Animal Sciences, Zhejiang University, Hangzhou 310058, P.R. China, ${ }^{2}$ Institute of Sericulture, Chengde Medical University, Chengde 067000, P.R. China. YC: Key Laboratory of Genome Sciences and Information, Beijing Institute of Genomics, Chinese Academy of Sciences, Beijing, 100029, China.

\section{Acknowledgement}

This work was supported by the grants from the National Basic Research Program of China (No. 2012CB114601), Projects of Zhejiang Provincial Science and Technology Plans (No.2013C32048 and No.2012C12910) and Project of Hebei Provincial Department of Education (No. Q2012026).

\section{Author details}

${ }^{1}$ College of Animal Sciences, Zhejiang University, Hangzhou, Hangzhou 310058, P.R. China. ${ }^{2}$ Institute of Sericulture, Chengde Medical University, Chengde 067000, P.R. China. ${ }^{3}$ Key Laboratory of Genome Sciences and Information, Beijing Institute of Genomics, Chinese Academy of Sciences, Beijing 100029, China.
Received: 19 December 2013 Accepted: 7 May 2014

Published: 29 May 2014

\section{Reference}

1. Goldsmith MR, Shimada T, Abe H: The genetics and genomics of the silkworm, bombyx mori*. Annu Rev Entomol 2005, 50:71-100.

2. Zhou Y: General Entomology. Beijing, China: High Education Publication House; 1958.

3. Zhang P, Aso Y, Yamamoto K, Banno Y, Wang Y, Tsuchida K, Kawaguchi Y, Fujii H: Proteome analysis of silk gland proteins from the silkworm, Bombyx mori. Proteomics 2006, 6:2586-2599.

4. Li J, Yang H, Lan T, Wei H, Zhang H, Chen M, Fan W, Ma Y, Zhong B: Expression profiling and regulation of genes related to silkworm posterior silk gland development and fibroin synthesis. J Proteome Res 2011, 10:3551-3564

5. Xia Q, Cheng D, Duan J, Wang G, Cheng T, Zha X, Liu C, Zhao P, Dai F, Zhang Z: Microarray-based gene expression profiles in multiple tissues of the domesticated silkworm. Bombyx mori Genome Biology 2007, 8:R162.

6. Niwa R, Slack FJ: The evolution of animal microRNA function. Curr Opin Genet Dev 2007, 17:145-150.

7. He L, Hannon GJ: MicroRNAs: small RNAs with a big role in gene regulation. Nat Rev Genet 2004, 5:522-531.

8. Chen K, Rajewsky N: The evolution of gene regulation by transcription factors and microRNAs. Nat Rev Genet 2007, 8:93-103.

9. Ambros V: The functions of animal microRNAs. Nature 2004, 431:350-355.

10. Bartel DP: MicroRNAs: genomics, biogenesis, mechanism, and function. Cell 2004, 116:281-297.

11. Bushati N, Cohen SM: microRNA functions. Annu Rev Cell Dev Biol 2007, 23:175-205.

12. Wang QL, Li ZH: The functions of microRNAs in plants. Front Biosci 2007, 12:3975-3982

13. Krol J, Loedige I, Filipowicz W: The widespread regulation of microRNA biogenesis, function and decay. Nat Rev Genet 2010, 11:597-610.

14. Friedman JM, Jones PA: MicroRNAs: critical mediators of differentiation, development and disease. Swiss Med Wkly 2009, 139:466.

15. Tong $C$, Jin $Y$, Zhang $Y$ : Computational prediction of microRNA genes in silkworm genome. J Zhejiang Univ Sci B 2006, 7:806-816.

16. Cao J, Tong C, Wu X, LV J, Yang Z, Jin Y: Identification of conserved microRNAs in Bombyx mori (silkworm) and regulation of fibroin $L$ chain production by microRNAs in heterologous system. Insect Biochem Mol Biol 2008, 38:1066-1071.

17. He P, Nie Z, Chen J, Lv Z, Sheng Q, Zhou S, Gao X, Kong L, Wu X: Identification and characteristics of microRNAs from Bombyx mori. BMC Genomics 2008, 9:248.

18. Huang Y, Zou Q, Tang SM, Wang LG, Shen XJ: Computational identification and characteristics of novel microRNAs from the silkworm (Bombyx mori L.). Mol Biol Rep 2010, 37:3171-3176.

19. Zhang Y, Zhou X, Ge X, Jiang J, Li M, Jia S, Yang X, Kan Y, Miao X, Zhao G: Insect-specific microRNA involved in the development of the silkworm Bombyx mori. PLoS One 2009, 4:e4677.

20. Cai Y, Yu X, Zhou Q, Yu C, Hu H, Liu J, Lin H, Yang J, Zhang B, Cui P: Novel microRNAs in silkworm (Bombyx mori). Funct Integr Genomics 2010, 10:405-415

21. Jagadeeswaran $G$, Zheng $Y$, Sumathipala N, Jiang $H$, Arrese EL, Soulages $J$, Zhang W, Sunkar R: Deep sequencing of small RNA libraries reveals dynamic regulation of conserved and novel microRNAs and microRNA-stars during silkworm development. BMC Genomics 2010, 11:52.

22. Liu S, Li D, Li Q, Zhao P, Xiang Z, Xia Q: MicroRNAs of Bombyx mori identified by Solexa sequencing. BMC Genomics 2010, 11:148.

23. Lagos-Quintana M, Rauhut R, Yalcin A, Meyer J, Lendeckel W, Tuschl T: Identification of tissue-specific microRNAs from mouse. Curr Biol 2002, 12:735-739.

24. Shamimuzzaman M, Vodkin L: Identification of soybean seed developmental stage-specific and tissue-specific miRNA targets by degradome sequencing. BMC Genomics 2012, 13:310.

25. Dudek KA, Lafont JE, Martinez-Sanchez A, Murphy CL: Type II collagen expression is regulated by tissue-specific miR-675 in human articular chondrocytes. J Biol Chem 2010, 285:24381-24387.

26. Tashiro Y, Morimoto T, Matsuura S, Nagata S: Studies on the posterior silk gland of the silkworm, Bombyx mori. J Cell Biol 1968, 38:574-588. 
27. Chen X, Li Q, Wang J, Guo X, Jiang X, Ren Z, Weng C, Sun G, Wang X, Liu Y: Identification and characterization of novel amphioxus microRNAs by Solexa sequencing. Genome Biol 2009, 10:R78

28. Barrett T, Troup DB, Wilhite SE, Ledoux P, Rudnev D, Evangelista C, Kim IF, Soboleva A, Tomashevsky M, Marshall KA: NCBI GEO: archive for high-throughput functional genomic data. Nucleic Acids Res 2009, 37:D885-D890.

29. Wei Y, Chen S, Yang P, Ma Z, Kang L: Characterization and comparative profiling of the small RNA transcriptomes in two phases of locust. Genome Biol 2009, 10:R6.

30. Liang C, Zhang X, Zou J, Xu D, Su F, Ye N: Identification of miRNA from Porphyra yezoensis by high-throughput sequencing and bioinformatics analysis. PLoS One 2010, 5:e10698.

31. Kozomara A, Griffiths-Jones S: miRBase: integrating microRNA annotation and deep-sequencing data. Nucleic Acids Res 2011, 39:D152-D157.

32. Huang PJ, Liu YC, Lee CC, Lin WC, Gan RR, Lyu PC, Tang P: DSAP: deep-sequencing small RNA analysis pipeline. Nucleic Acids Res 2010 38:W385-W391.

33. Williamson V, Kim A, Xie B, McMichael GO, Gao Y, Vladimirov V: Detecting miRNAs in deep-sequencing data: a software performance comparison and evaluation. Brief Bioinform 2012, 14:36-45.

34. Li M, Xia Y, Gu Y, Zhang K, Lang Q, Chen L, Guan J, Luo Z, Chen H, Li Y: MicroRNAome of porcine pre-and postnatal development. PLoS One 2010, 5:e11541.

35. Xie SS, Li XY, Liu T, Cao JH, Zhong Q, Zhao SH: Discovery of porcine microRNAs in multiple tissues by a Solexa deep sequencing approach. PLoS One 2011, 6:e16235.

36. Lian C, Sun B, Niu S, Yang R, Liu B, Lu C, Meng J, Qiu Z, Zhang L, Zhao Z: A comparative profile of the microRNA transcriptome in immature and mature porcine testes using Solexa deep sequencing. FEBS J 2012, 279:964-975.

37. MIREAP. https://sourceforge.net/projects/mireap/.

38. Liu X, He S, Skogerbo G, Gong F, Chen R: Integrated Sequence-Structure Motifs Suffice to Identify microRNA Precursors. PLoS One 2012, 7:e32797.

39. Xue C, Li F, He T, Liu GP, Li Y, Zhang X: Classification of real and pseudo microRNA precursors using local structure-sequence features and support vector machine. BMC Bioinforma 2005, 6:310.

40. Zhao D, Wang Y, Luo D, Shi X, Wang L, Xu D, Yu J, Liang Y: PMirP: A pre-microRNA prediction method based on structure-sequence hybrid features. Artif Intell Med 2010, 49:127-132.

41. Li J, FAN W, YOU Z, ZHONG B: Bioinformatics analysis on structural features of microRNA precursors in insects. Eur J Entomol 2013, 110:13-20.

42. Liu S, Zhang L, Li Q, Zhao P, Duan J, Cheng D, Xiang Z, Xia Q: MicroRNA expression profiling during the life cycle of the silkworm (Bombyx mori). BMC Genomics 2009, 10:455.

43. Willenbrock H, Salomon J, Søkilde R, Barken KB, Hansen TN, Nielsen FC, Møller S, Litman T: Quantitative miRNA expression analysis: comparing microarrays with next-generation sequencing. RNA 2009, 15:2028-2034.

44. Barad O, Meiri E, Avniel A, Aharonov R, Barzilai A, Bentwich I, Einav U, Gilad $S$, Hurban P, Karov Y: MicroRNA expression detected by oligonucleotide microarrays: system establishment and expression profiling in human tissues. Genome Res 2004, 14:2486-2494.

45. Liu C-G, Calin GA, Volinia S, Croce CM: MicroRNA expression profiling using microarrays. Nat Protoc 2008, 3:563-578.

46. Liu S, Gao S, Zhang D, Yin J, Xiang Z, Xia Q: MicroRNAs show diverse and dynamic expression patterns in multiple tissues of Bombyx mori. BMC Genomics 2010, 11:85.

47. Wienholds E, Kloosterman WP, Miska E, Alvarez-Saavedra E, Berezikov E, de Bruijn E, Horvitz HR, Kauppinen S, Plasterk RH: MicroRNA expression in zebrafish embryonic development. Science 2005, 309:310-311.

48. Enright AJ, John B, Gaul U, Tuschl T, Sander C, Marks DS: MicroRNA targets in Drosophila. Genome Biol 2004, 5:R1-R1.

49. Zhou ZH, Yang HJ, Chen M, Lou CF, Zhang YZ, Chen KP, Wang Y, Yu ML, Yu F, Li JY, Zhong BX: Comparative proteomic analysis between the domesticated silkworm (Bombyx mori) reared on fresh mulberry leaves and on artificial diet. J Proteome Res 2008, 7:5103-5111.

50. Li J, Ye L, Lan T, Yu M, Liang J, Zhong B: Comparative proteomic and phosphoproteomic analysis of the silkworm (Bombyx mori) posterior silk gland under high temperature treatment. Mol Biol Rep 2012, 39:8447-8456.
51. Zhong B, Yu Y, Xu Y, Yu H, Lu X, Miao Y, Yang J, Xu H, Hu S, Lou C: EST Sequencing and Gene Expression Profiling Analysis of the Posterior Silk Gland Cells of the Fifth Instar Larvae of Silkworm. Science In China Ser C Life Sci 2004, 34:436-443.

52. Ciechanover A, Orian A, Schwartz AL: Ubiquitin-mediated proteolysis: biological regulation via destruction. Bioessays 2000, 22:442-451.

53. Tanaka T: The anatomy of the silkworm. Tokyo: Meibun-Do Co; 1928.

54. Wang X, Zhang J, Li F, Gu J, He T, Zhang X, Li Y: MicroRNA identification based on sequence and structure alignment. Bioinformatics 2005, 21:3610-3614

55. Glazov EA, Cottee PA, Barris WC, Moore RJ, Dalrymple BP, Tizard ML: A microRNA catalog of the developing chicken embryo identified by a deep sequencing approach. Genome Res 2008, 18:957-964.

56. Hafner M, Landgraf P, Ludwig J, Rice A, Ojo T, Lin C, Holoch D, Lim C, Tuschl T: Identification of microRNAs and other small regulatory RNAs using cDNA library sequencing. Methods 2008, 44:3-12.

57. Zhu Q, Hong A, Sheng N, Zhang X, Matejko A, Jun K, Srivannavit O, Gulari E, Gao X, Zhou X: $\mu$ Paraflo ${ }^{\text {TM }}$ Biochip for Nucleic Acid and Protein Analysis. Methods Mol Biol-Clifton Then Totowa- 2007, 382:287.

58. Bolstad BM, Irizarry RA, Åstrand M, Speed TP: A comparison of normalization methods for high density oligonucleotide array data based on variance and bias. Bioinformatics 2003, 19:185-193.

doi:10.1186/1471-2164-15-410

Cite this article as: Li et al:: MicroRNA expression profiling of the fifthinstar posterior silk gland of Bombyx mori. BMC Genomics 2014 15:410.

\section{Submit your next manuscript to BioMed Central and take full advantage of:}

- Convenient online submission

- Thorough peer review

- No space constraints or color figure charges

- Immediate publication on acceptance

- Inclusion in PubMed, CAS, Scopus and Google Scholar

- Research which is freely available for redistribution 